\title{
Dinámica poblacional de Ceroxylon quindiuense (H.Karst.) H.Wendl. (Arecaceae) a partir de matrices de Leslie como herramienta de conservación. Salento, Colombia
}

\author{
Population dynamics of Ceroxylon quindiuense (H.Karst.) H.Wendl. \\ (Arecaceae) from Leslie matrix as a conservation tool. Salento, Colombia
}

\author{
Juan Carlos Álvarez-Londoño**, Lina Marcela Ocampo*, Janeth Molina*
}

Resumen

\begin{abstract}
El género Ceroxylon es endémico de las montañas Andinas y es uno de los más importantes de esta región.Incluye 11 especies distribuidas desde los $800 \mathrm{~m}$ hasta los $3500 \mathrm{~m}$ de altitud y a menudo son árboles dominantes del dosel. Para la evaluación poblacional de Ceroxylon quindiuense (H.Karst.) H.Wendl. en la hacienda La Palmera se trazaron tres transectos cada uno de 150 metros, donde se establecieron 16 puntos, separados cada $10 \mathrm{~m}$ entre sí. Se seleccionaron la mitad de los puntos que correspondían a los impares, en cada uno se ubicó el individuo más cercano al punto y se midió la distancia del punto a la planta. En la mitad restante de los puntos de muestreo se determinó la distancia del individuo al vecino más cercano; para estimar la distribución espacial se utilizó la prueba de Hopkins (1954), además con la distribución de los individuos por edad se utilizó el modelo matricial de Leslie para la construcción del modelo en el tiempo. La simulación poblacional se obtuvo mediante el software matemático MATLAB versión 6.1, se empleo la tasa de supervivencia real para C. quindiuense modificándola $15 \%$ por encima y $15 \%$ por debajo, con esto se logró determinar que $C$. quindiuense es altamente vulnerable por su largo ciclo de vida, lenta germinación y lento crecimiento. El mayor índice de mortalidad se observa en el cambio de estadío plántulas-jóvenes.
\end{abstract}

Palabras clave: Ceroxylon quindiuense, Conservación, Modelo poblacional, Simulaciones.

\begin{abstract}
Ceroxylon is a endemic genus to the Andes mountains and one of the most important in this region, includes 11 species distributed from 800-3500 m above sea level and are often dominant canopy trees. For the evaluation of Ceroxylon quindiuense (H.Karst.) H.Wendl. population to the Hacienda La Palmera were drawn three transects each 150 meters, which were established in 16 point. All points were 10 $\mathrm{m}$ apart from each other. Were selected points corresponding to half odd points in each odd point was located nearest to the individual point distance and measured point to the plant. With the distribution of individuals by age was construct the model in time used Leslie matrix The simulated population was obtained by the mathematical software MATLAB version 6.1, use the actual survival rate for $C$. quindiuense modifying $15 \%$ above and $15 \%$ below, with this we determine that $C$. quindiuense is highly vulnerable because of its long life cycle, slow germination and slow growth. The increased mortality rate is observed in the change-young seedling stage to juvenile plant.
\end{abstract}

Keywords: Ceroxylon quindiuense, Conservation, Population dynamics, Simulations.

\section{Introducción}

Las poblaciones varían a través del tiempo por medio de procesos continuos o discretos, estos reci- ben el nombre de dinámica poblacional. Moraes et al. (1995) y Rodríguez et al. (2005) definen que el principal objetivo de la dinámica de poblaciones es estudiar sus cambios numéricos, determinar las cau-

Escuela de Investigación en Biomatemática, Universidad del Quindío, Armenia, Colombia. e-mail: ayoira21@gmail.com shelita0917783@gmail.com janneth@uniquindio.edu.co Fecha recepción: Noviembre 29, $2012 \quad$ Fecha aprobación: Enero 7, 2013 
sas de esta variación, predecir su comportamiento a futuro y analizar las consecuencias ecológicas que esto produce para promover estrategias de conservación. Las Matrices de Leslie se utilizan en ecología porque permiten describir el crecimiento de una población en función del tiempo, utilizando una clasificación por estadíos según la edad (Alvarez-Buylla 1994, Barot 1994); a partir de las simulaciones arrojadas se puede observar el comportamiento en el tiempo y la vulnerabilidad de la población según la edad, por lo que es posible contribuir a la toma de decisiones sobre el manejo de la población para garantizar su continuidad según los recursos de los que dispone en cada estadío.

El estudio demográfico es de gran importancia para la conservación de poblaciones silvestres cuyo número está en descenso o que están en riesgo de extinción (Harper 1974, Rodríguez et al. 2005), en estos casos es donde los análisis matriciales son fundamentales al momento de realizar estudios demográficos pues proporcionan información adecuada para visualizar como es el flujo numérico a través del tiempo; según Horvitz y Schemeske (1995), estudiar las causas de este flujo numérico puede ayudar a determinar los mecanismos que regula la población en términos ecológicos y evolutivos, siendo esto fundamental en los procesos de conservación (Harper 1977, Olmsted y Álvarez-Buylla 1995). El conocimiento de la distribución de una especie puede dar una idea acerca del comportamiento de la población y permite realizar inferencias acerca de cómo puede ir cambiando a través del tiempo y frente a distintas prácticas de manejo (Galeano y Bernal 2002), donde el análisis matricial es de gran ayuda en estos procesos de conservación en palmas (Olmsted y Álvarez-Buylla 1995, Álvarez-Buylla 1994, Barot 1994).

Las palmeras (familia Arecaceae) constituyen un componente muy importante en el dosel del bosque montano. Un ejemplo clásico del dominio de palmas en bosque montano es tal vez la especie colombiana Ceroxylon quindiuense (H.Karst.) H.Wendl. que cuenta con una legendaria reputación entre los primeros exploradores europeos del norte de los Andes debido a su espectacular altura, troncos blancos y poblaciones densas (Borchsenius y Moraes 2006). El género Ceroxylon es endémico de las montañas Andinas y uno de los más importantes de esta región. El género incluye 11 especies (Galeano 1995, Henderson et al. 1996) distribuidas entre 800 y 3500 $\mathrm{m}$ de altitud. Varias especies tienen rangos geográficos estrechos y algunas poblaciones son sometidas a un elevado grado de fragmentación debido a la deforestación y conversión de tierras agrícolas en sus hábitats (Borchsenius y Moraes 2006).

Las palmas tienen una relevante importancia en la conformación de las comunidades de los bosques tropicales. Ellas pueden ejercer gran influencia en las condiciones medio ambientales del sotobosque, en donde posteriormente se establecerá la gran mayoría de las especies del bosque (Pintaud et al. 2010). La presencia de las palmas influye sobre la diversidad del sitio específico donde éstas se encuentran, sus frutos representan una fuente importante de alimento para varios grupos de animales frugívoros y requieren por lo general de insectos, aves o mamíferos para la polinización y diseminación de sus semillas; además, las palmas aportan un alto contenido de hojarasca al suelo del bosque, lo que evidencia la participación de estas plantas en el ciclo de nutrientes lo que constituye un aporte fundamental en la dinámica del ecosistema (Girón 2001).

Las palmas de cera son habitantes típicos del bosque primario andino y solo se reproducen bajo las condiciones de sombra y humedad existentes en él. Cuando el bosque natural es derribado para establecer áreas de potreros o cultivos, las poblaciones de palma son severamente diezmadas, poniéndolas en inminente peligro de extinción. Si se continúa sobreexplotando los bosques de palma que albergan esta especie, será muy difícil que estos logren permanecer (Pintaud et al. 2010). La palma de cera del Quindío C. quindiuense crece por encima de los 2000 msnm, normalmente alcanza $60 \mathrm{~m}$, pudiendo llegar hasta $80 \mathrm{~m}$ por las asociaciones de C.quinduense que son relativamente estables con respecto a la estructura y composición florística establecidas por las especies comunes en el área. La regeneración natural de los bosques es muy lenta en áreas donde hay intervención antrópica (Nieto y Rodríguez 2002). Además, la cera que se produce en los troncos y hojas, fue muy utilizada comercialmente para la producción de antorchas y velas, y para eso era necesario tumbar las palmas lo que diezmaba la población (Borchsenius y Moraes 2006).

En esta investigación se pretende conocer el pa- 
trón de distribución, la densidad y el estado poblacional de $C$. quindiuense en el tiempo, utilizando las Matrices de Leslie para generar posibles estrategias de conservación de acuerdo con las categorías de edad de la población.

\section{Metodología}

Área de estudio. La reserva natural La CabañaLa Esperanza, con unas coordenadas geográficas de latitud N: $4^{\circ} 39^{\prime}$ latitud W: $75^{\circ} 37^{\prime}$, se localiza en el flanco occidental de la cordillera Central, en la vereda Cocora, municipio de Salento, departamento del Quindío, en la cuenca alta del río Quindío (Figura 1). Esta reserva se ubica en un rango altitudinal entre los 2300 y $3750 \mathrm{~m}$ de elevación, con áreas de ladera que comprenden desde la base hasta las cimas de montaña como la zona amortiguadora del Parque Nacional Natural de los Nevados. La precipitación promedio anual es de $1820.67 \mathrm{~mm}$ y la temperatura fluctúa entre $8^{\circ}$ y $22^{\circ} \mathrm{C}$. La reserva tiene un área total de 565 ha, distribuidas en diferentes franjas vegetales o ecosistemas según su rango altitudinal y composición florística.
Densidad y distribución. Para determinar el patrón de distribución de las poblaciones de $C$. quindiuense se siguió el método de muestreo propuesto por Byth y Ripley (1980), que consiste en trazar un transecto de $140 \mathrm{~m}$, donde se establecieron 14 puntos. Por último, para la población de $C$. quindiuense de la hacienda La Palmera, se trazaron tres transectos cada uno de $150 \mathrm{~m}$, en los cuales se establecieron 16 puntos. Todos los puntos eran separados por 10 m entre sí. De los puntos se seleccionaron la mitad que correspondían a puntos impares, en cada punto impar se ubicó el individuo más cercano al punto y se midió la distancia del punto al organismo. En la mitad restante de los puntos de muestreo, puntos pares, se ubicó el individuo más cercano a cada punto, después se midió la distancia entre este y su vecino más cercano.

Para estimar la distribución espacial se utilizó la prueba de Hopkins (1954) la cual es también utilizada por Krebs (1978). Para procesar los datos colectados en campo la prueba aparece a continuación:

$$
\mathrm{h}=\Sigma\left(\mathrm{x}_{\mathrm{i}}^{2}\right) / \Sigma\left(\mathrm{r}_{\mathrm{i}}^{2}\right)
$$

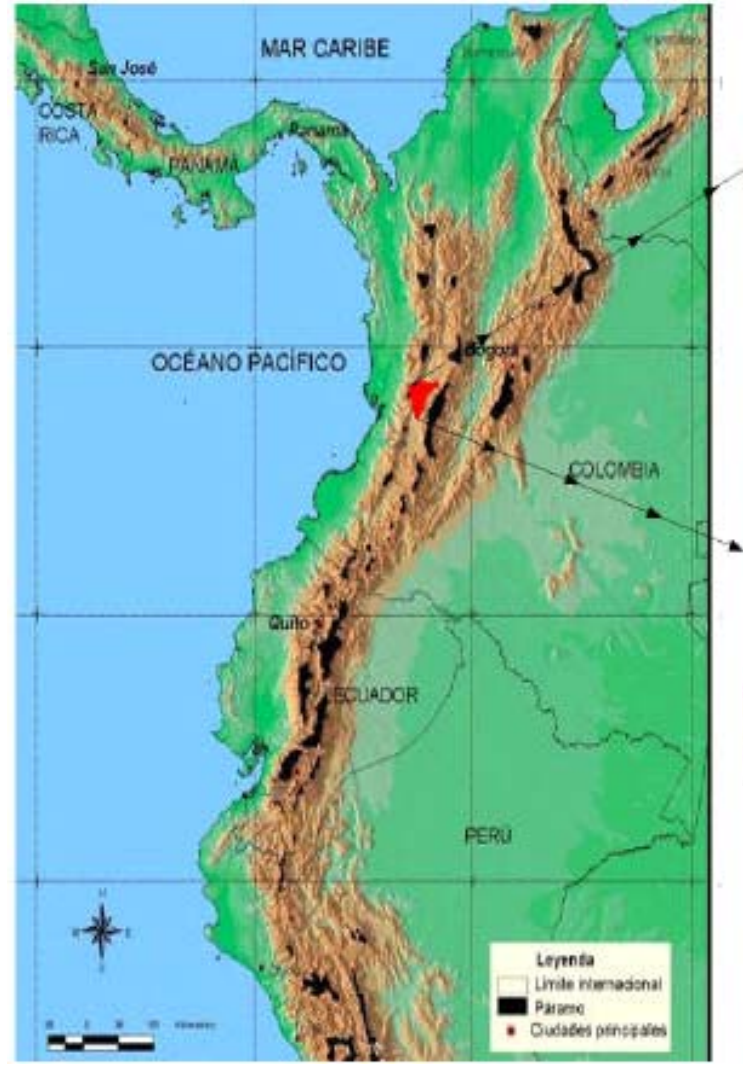

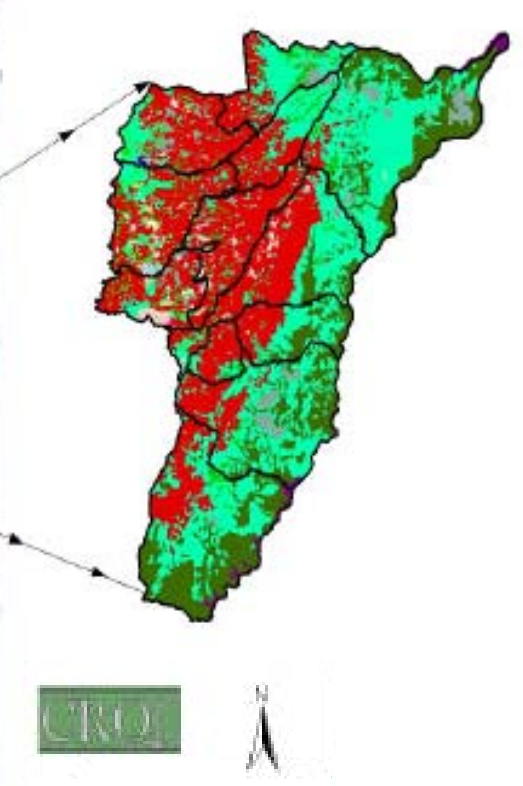

Figura 1. Localización del departamento del Quindío. El punto negro sobre el departamento del Quindío indica la zona de estudio «reserva natural La Cabaña».

Imagen suministrada por la Corporación Autónoma del Quindío. 
donde:

$h$ es la prueba estadística de Hopkins para determinar el patrón espacial al azar

$\mathrm{x}_{\mathrm{i}}$ es la distancia desde el punto al azar $i$ al organismo más cercano

$r_{i}$ es la distancia del individuo al azar $i$ al vecino más cercano

Hopkins demostró que $h$ está distribuido como $\mathrm{F}$ con $2 \mathrm{n}$ grados de libertad en el numerador y lo mismo en el denominador. La justificación intuitiva de la prueba de Hopkins es que si los organismos están agrupados, la distancia punto a organismo sería más grande en relación con la distancia organismo a organismo. Lo opuesto ocurriría si el patrón espacial fuera uniforme. Así, la prueba $\mathrm{F}$ para $h$ es una prueba de dos colas donde $h$ sería significativamente pequeña si existe uniformidad.

El rango para estimar la distribución está desde 0 y 1 y se estima de la siguiente manera:

$$
\mathrm{I}_{\mathrm{H}}=\mathrm{h} /(1+\mathrm{h})
$$

Este índice se aproxima a 1 cuando incrementa el agrupamiento y se aproxima a cero cuando la uniformidad es máxima. Cuando el patrón es al azar el índice sería 0.5 (Gilbert et al. 1994).

Para la estimación de la densidad en todos los métodos de distancia es sensible al patrón espacial. Si los individuos muestreados tienen un patrón espacial al azar, todas las medidas de distancia suministran una estimación no sesgada de la densidad de población. Por esto, es importante determinar el patrón espacial antes de poder estimar la densidad cuando se utiliza el método de distancia (Pacala et al. 1996, Camarero y Rozas 2006).

Si la población estudiada presenta una distribución aleatoria, cualquier medida de distancia sugerida por Byth y Ripley (1980) suministra una estimación no sesgada de la densidad de población. Para distancia punto-organismo se tiene:

$$
\mathrm{N}_{1}=\mathrm{n} /\left(\pi \Sigma\left(\mathrm{x}_{\mathrm{i}}^{2}\right)\right.
$$

donde:

$\mathrm{N}_{1}$ es la estimación de la densidad de población $n$ es el tamaño de la muestra

$\mathrm{x}_{\mathrm{i}}$ es la distancia del punto $i$ al organismo más cercano
Para distancia organismo-vecino más cercano se tiene:

$$
\mathrm{N}_{2}=\mathrm{n} /\left[\pi \Sigma\left(\mathrm{r}_{\mathrm{i}}^{2}\right)\right]
$$

donde:

$\mathrm{N}_{2}$ es la estimación de la densidad de población $r_{i}$ es la distancia del individuo $i$ a su vecino más cercano

Las varianzas de estos estimadores son similares y se pueden usar para poner límites de confidencia en la densidad estimada. La varianza se estima del recíproco de la densidad, así:

$$
\begin{gathered}
\mathrm{y}=1 / \mathrm{N} \\
\operatorname{varianza} d e(y)=y^{2} / \mathrm{n} \text { Error estándar }(\mathrm{y})= \\
\operatorname{varianza}(\mathrm{y}) / \mathrm{n}
\end{gathered}
$$

donde:

$n$ es el tamaño de la muestra

$\mathrm{N}$ puede ser $\mathrm{N}_{1}$ o $\mathrm{N}_{2}$

(Krebs 1978, Pacala et al. 1996)

Demografía. El análisis de la dinámica poblacional de $C$. quindiuense se realizó en la hacienda La Palmera. Para la toma de datos se realizaron 2 parcelas de $150 \mathrm{~m} \times 5 \mathrm{~m}$, ubicada sobre los transectos utilizados para analizar la densidad y distribución espacial; estas se dividieron en subparcelas de $10 \mathrm{~m} \times 5 \mathrm{~m}$. En cada una de las subparcelas se registraron todos los individuos de C. quindiuense clasificándolos según la categoría de edad; también se tomó la altura y el número de hojas por individuo. La categoría de edad se asignó de acuerdo con los siguientes parámetros:

- 0 a $50 \mathrm{~cm}$ plántulas

- $50 \mathrm{~cm}$ a $5 \mathrm{~m}$ jóvenes

- $>5 \mathrm{~m}$ adultos

Estos datos son tomados de la tabla de vida obtenida por Girón et al. (2001).

Para determinar el crecimiento de la población de C. quindiuense de la hacienda La Palmera, así como la distribución por edad a lo largo del tiempo, se utilizó el modelo matricial de Leslie (Figura 2). La simulación poblacional se obtuvo mediante el software matemático MATLAB versión 6.1. La simulación fue hecha para 50, 100 y 150 años con el empleo de la tasa de supervivencia real para 


$$
\left(\begin{array}{c}
x_{1}^{(k)} \\
x_{2}^{(k)} \\
x_{3}^{(k)} \\
\vdots \\
x_{n}^{(k)}
\end{array}\right)=\left(\begin{array}{cccccc}
F_{1} & F_{2} & F_{3} & \ldots & F_{n-1} & F_{n} \\
P_{1} & 0 & 0 & \ldots & 0 & 0 \\
0 & P_{2} & 0 & \ldots & 0 & 0 \\
\vdots & \vdots & \vdots & & \vdots & \vdots \\
0 & 0 & 0 & \ldots & P_{n-1} & 0
\end{array}\right) \quad\left(\begin{array}{c}
x_{1}^{(k-1)} \\
x_{2}^{(k-1)} \\
x_{3}^{(k-1)} \\
\vdots \\
x_{n}^{(k-1)}
\end{array}\right)
$$

Figura 2. Modelo matricial de Leslie utilizado en el programa MatLab 6.1 para hallar la distribución por edad a lo largo del tiempo. Donde $\mathrm{F}_{1}$ es la tasa de reproducción, $\mathrm{P}_{1}$ es la probabilidad de supervivencia.

C. quindiuense (Girón 2000) y modificándola $15 \%$ por encima y $15 \%$ por debajo de ésta.

\section{Resultados}

Distribución y densidad. La población de C. quindiuense presenta un patrón de distribución al azar pues el valor que arrojó el índice fue de 0,494. La densidad poblacional de $C$. quindiuense en la reserva natural La Cabaña-La Esperanza es de 0,0153 individuos $/ \mathrm{m}^{2}$. La densidad poblacional de $C$. quindiuense del lugar de muestreo transecto 1 del bosque La Palmera es de $0,751 \mathrm{palma} / \mathrm{m}^{2}$ si se basa en las distancias tomadas del punto al individuo más cercano y/o 1,112 palma $/ \mathrm{m}^{2}$ si se basa en las distancias tomadas de individuo al vecino más cercano. Para el lugar de muestreo 2 en la hacienda La Palmera la densidad poblacional de C. quindiuense es de 1,012 individuos $/ \mathrm{m}^{2}$ si se basa en las distancias tomadas del punto al individuo más cercano y/o 0,785 individuos $/ \mathrm{m}^{2}$ si se basa en las distancias tomadas de individuo a vecino más cercano.

Uniendo la población del transecto1 y la población del transecto 2 se obtuvo que la densidad poblacional de $C$. quindiuense del bosque el palmar en la hacienda La Palmera es de $0,911 \mathrm{palma} / \mathrm{m}^{2}$ si se basa en las distancias tomadas del punto al individuo más cercano y/o 0,888 palma $/ \mathrm{m}^{2}$ si se basa en las distan- cias tomadas de individuo a vecino más cercano.

Dinámica poblacional. Para el análisis de la dinámica poblacional se encontró que teniendo en cuenta el número de individuos en los tres estadíos (plán-tulas $=639$; jóvenes $=140$; adultos $=11$ ), con una tasa reproductiva de 0.459 , con una probabilidad de supervivencia de plántulas a jóvenes de 0.2208 , de jóvenes a adultos de 0.0786, y de 1 para la sobrevivencia de adultos (Matriz de Leslie, Figura 3). Al hacer simulaciones se encontró para las plántulas que la población desciende drásticamente a 5 individuos durante el primer año, para después aumentar hasta llegar a 21 en 50 años, 31 en 100 años y 46 en 150 años. En el caso de los jóvenes, el número de individuos cae abruptamente a partir del segundo año a un individuo, después de este período, la población de jóvenes se mantiene diezmada 4 en 50 años; 7 en 100 años; 10 en 150 años. La población de adultos aumenta desde el primer año hasta llegar a 47 en 50 años, 69 en 100 años y 101 individuos en 150 años. En la Figura 4, se muestran las gráficas obtenidas para los anteriores resultados en 50 y 150 años.

De acuerdo con las probabilidades obtenidas, se halló $15 \%$ de dichos valores y se le sumaron y restaron a los datos, dando como resultado una tasa reproductiva $0.5278(+15 \%)$ y $0.3901(-15 \%)$; probabilidad de supervivencia de plántulas a jóvenes

$$
\left(\begin{array}{l}
X_{1}^{A} \\
X_{2}^{A} \\
X_{3}^{A}
\end{array}\right)=\left(\begin{array}{ccc}
0 & 0 & 0.459 \\
0.219 & 0 & 0 \\
0 & 0.078 & 1
\end{array}\right)\left(\begin{array}{c}
639 \\
140 \\
11
\end{array}\right)
$$

Figura 3. Parámetros empleados en el modelo matricial de Leslie utilizado en el programa Matlab 6.1 para hallar la distribución por edad a lo largo del tiempo. Donde A son los años $(50,100,150)$. 

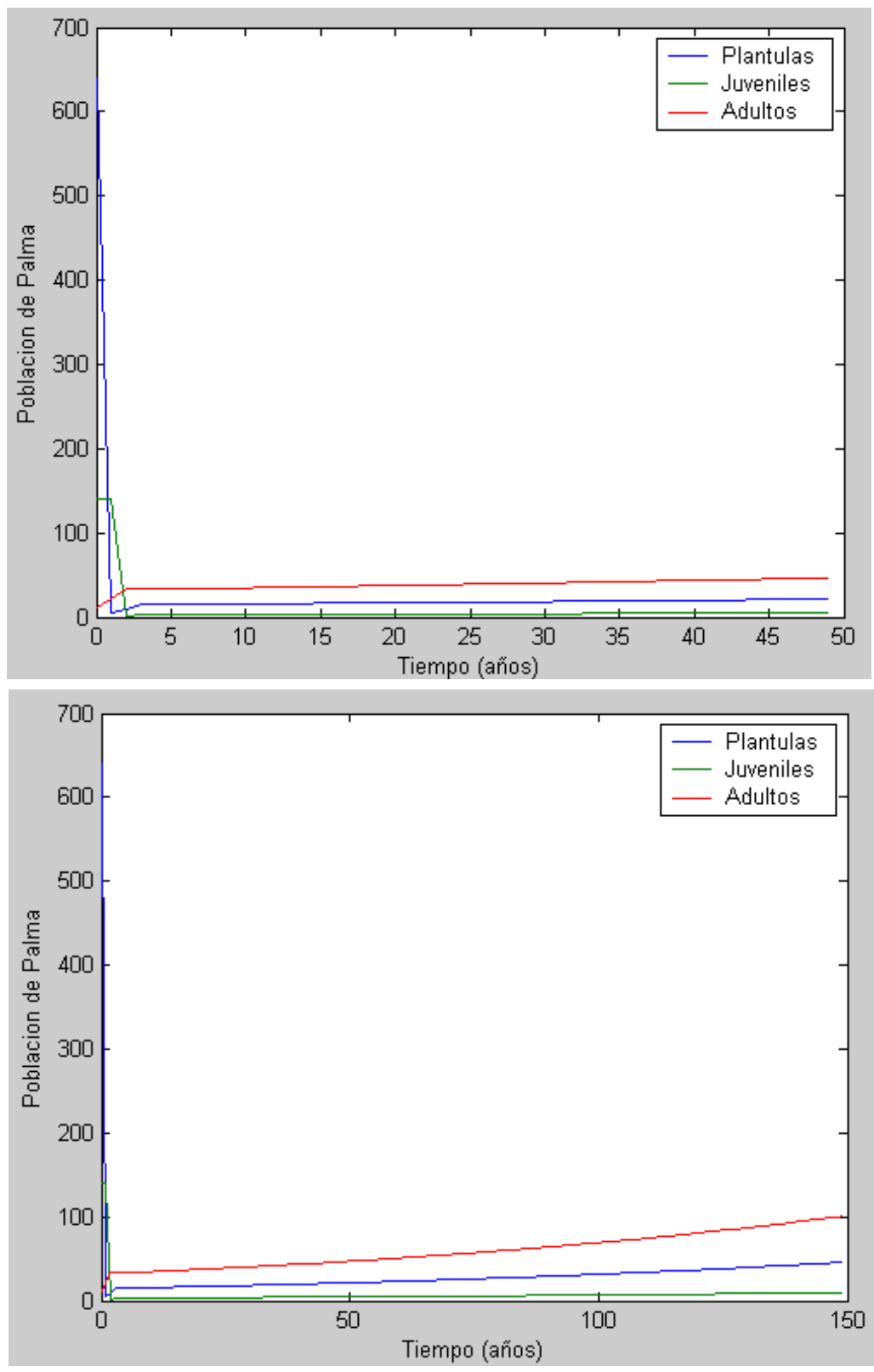

Figura 4. Gráficas de simulación poblacional de C. quindiuense, donde se evidencia el comportamiento de la población de la Hacienda La Palmera en la el municipio de Salento y la variación numérica de la población, en cada una de las tres categorías de edad (plántulas, jóvenes y adultos), 50 y 150 años respectivamente con los datos reales.

$0.2539(+15 \%)$ y $0.1877(-15 \%)$; jóvenes a adultos $0.0904(+15 \%)$ y $0.0681(-15 \%)$; la probabilidad de permanencia como adultos se mantuvo en 1 .

Con la sustracción del 15\% sobre las probabilidades se encontró que en 50 años el número de individuos en cada población será: plántulas 13, jóvenes 2 y adultos 35; en 100 años: plántulas 17 , jóvenes 3 y adultos 44 ; y en 150 años: plántulas 21 , jóvenes 4 y adultos 56. Las gráficas para 50 y 150 años se encuentran en la Figura 5.

En el caso en que las probabilidades se aumentaron en $15 \%$, en 50 años las poblaciones se espera que se encuentren así: plántulas 33, jóvenes 8 y adultos 64; en 100 años: plántulas 59, jóvenes 14 y adultos 114; y en 150 años: plántulas 105, jóvenes 26 y adultos 202 respectivamente (Figura 6). 

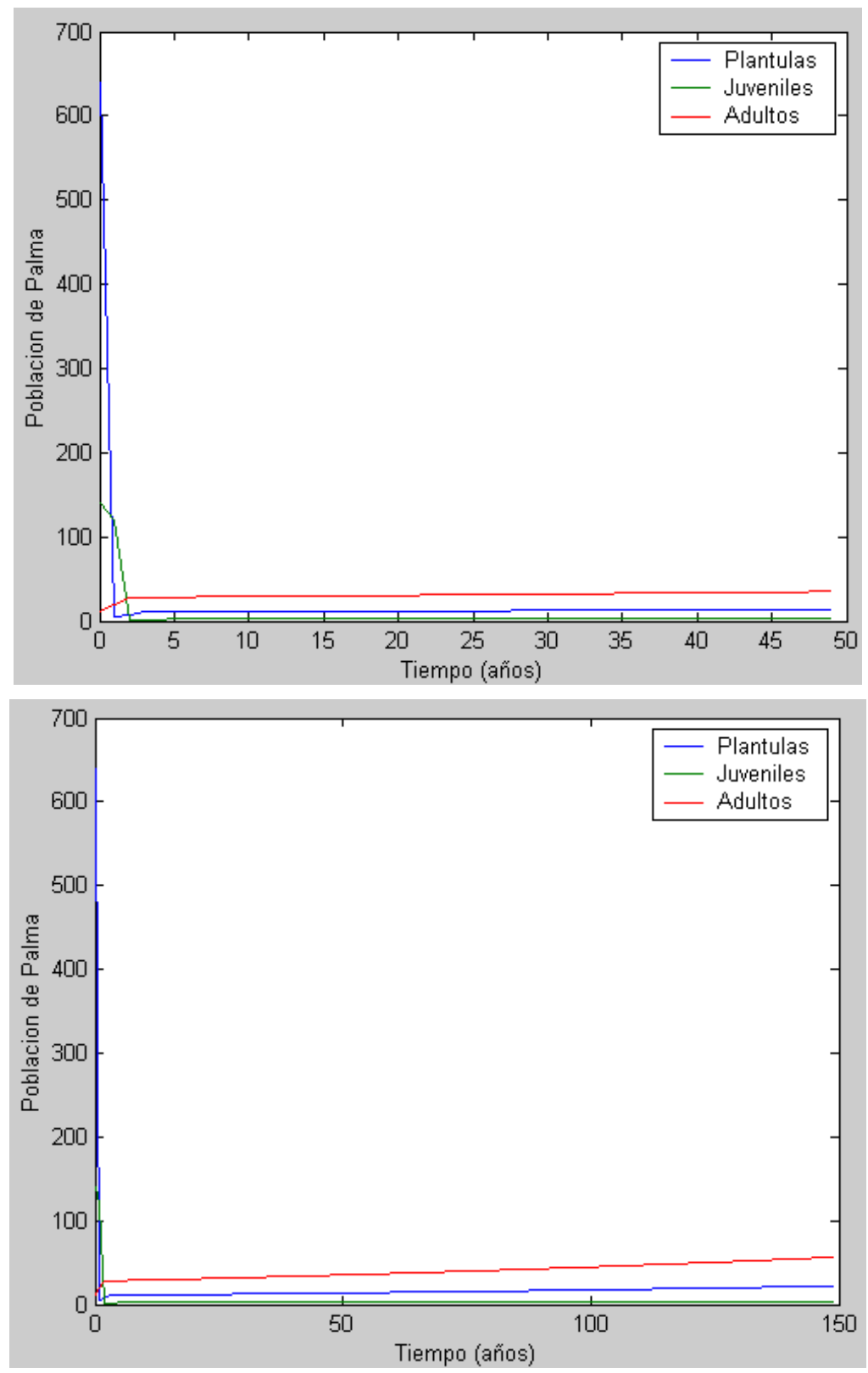

Figura 5. Gráficas de simulación poblacional de C. quindiuense, donde se muestra el comportamiento de la población de la Hacienda La Palmera y la variación numérica de la población, en cada una de las tres categorías de edad (plántulas, jóvenes y adultos). Las gráficas (50 años y 150 años) muestran una variación del 15\% por debajo de los datos reales de las tasas de reproducción y de supervivencia de plántulas y jóvenes.

\section{Discusión}

La tendencia de la población de C. quindiuense es hacia un patrón de distribución espacial al azar. En las plantas en general, es un principio ecológico bien definido el hecho de que la aglomeración o agrupación se relacione inversamente con la movilidad de las semillas (Odum 1986). Para las plantas que se reproducen por semillas, si la dispersión es a corta distancia en los individuos más jóvenes puede darse un patrón de distribución en manchones, aunque luego, debido a la eliminación por competencia intraespecífica el patrón tiende a ser aleatorio o uniforme (Matteucci y Colma 1982). En el caso de la palma de cera C. quindiuense, cuyas semillas no son móviles por sí mismas, hay una tendencia a encon- 

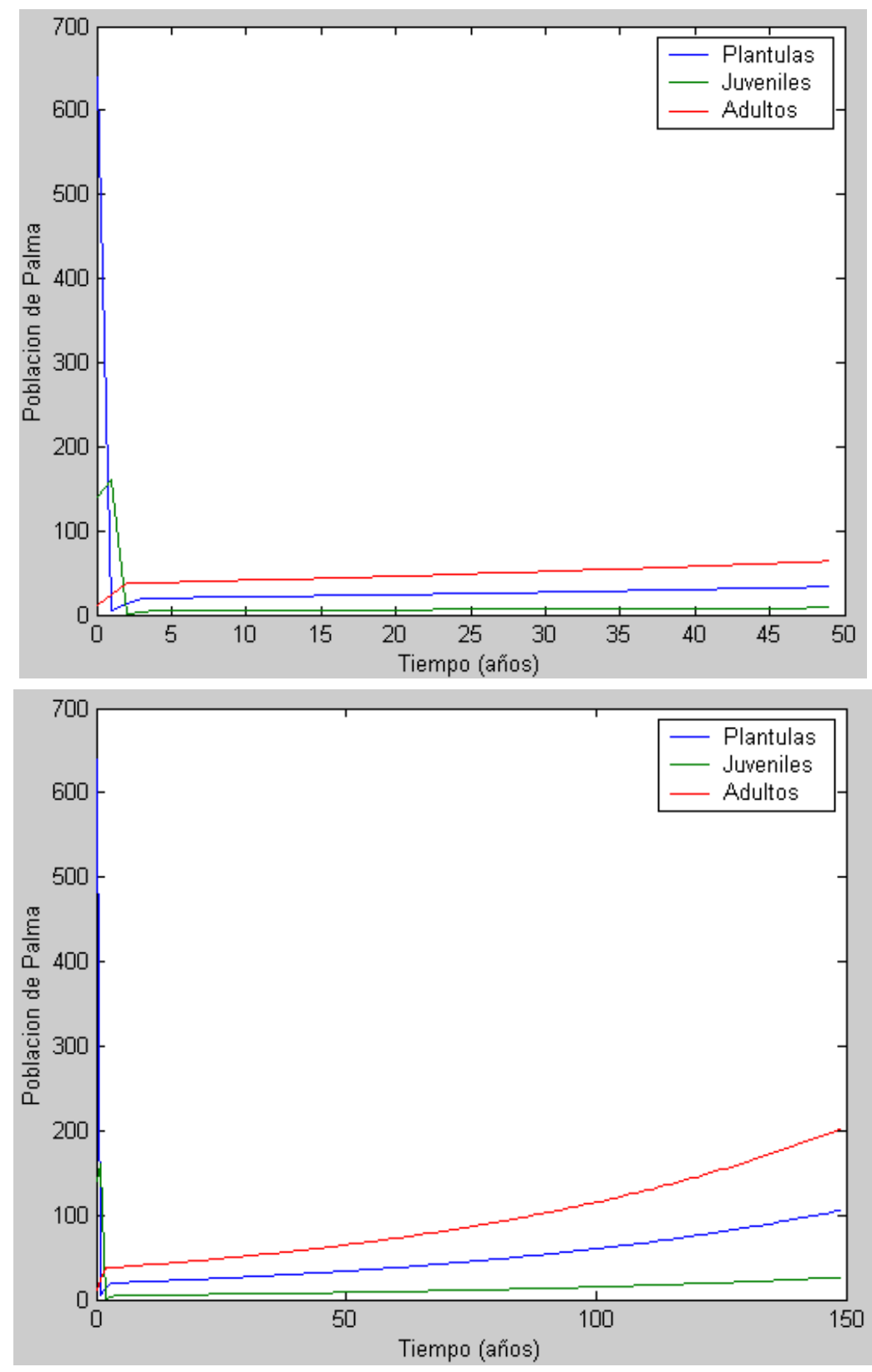

Figura 6. Gráficas de simulación poblacional de $C$. quindiuense, donde se muestra el comportamiento de la población de la Hacienda La Palmera y la variación numérica de la población, en cada una de las tres categorías de edad (plántulas, jóvenes y adultos). Variación del 15\% por encima de los datos reales de las tasas de reproducción y de supervivencia de plántulas y jóvenes.

trar plantas aglomeradas alrededor de los progenitores o en sitios donde las aves y otros animales han depositado las semillas en grupos (Girón y Arboleda 2001).

Según Odum (1986), el valor de la supervivencia del grupo de plantas de palma de cera es una característica importante que puede ser el resultado de la aglomeración. Esto quiere decir que grupo de plantas resiste mejor la acción del viento que los indivi- duos aislados y es capaz de reducir con mayor eficacia la pérdida de agua. No obstante en el caso de las plantas, los efectos nocivos de la competencia por la luz y los nutrientes suelen pesar más que las ventajas del agrupamiento (Galeano et al. 2008).

Como los individuos en el estadío de plántulas crecen agrupados alrededor del parental, pero, posiblemente por efectos de competencia entre ellos, se 
produce un mecanismo de selección en virtud del cual solo sobreviven los mejores adaptados, se encuentra una tendencia hacia un patrón de distribución al azar. En general, los factores que pueden influir en que la población de C. quindiuense presente un patrón espacial al azar, pueden tener diferentes orígenes: de tipo biótico como competencia, efectos de herbivoría, ataque de plagas; de tipo abiótico como la humedad relativa y la intensidad lumínica, que están variando continuamente a lo largo del día en el interior del bosque (Girón y Rodríguez 2001).

Según Spurr y Barnes (1980), la naturaleza y la cantidad de radiación solar recibida por el suelo del bosque afectarán la distribución, crecimiento y supervivencia de las plantas. Un factor que puede influir en el grado de agrupamiento de los individuos es la formación de claros en el bosque, y si estos aparecen al azar en un área del bosque, se podría explicar el patrón de distribución al azar de una determinada especie (Oliveira-Filho et al. 1998, Moraes 1999).

Otro factor que puede influir en la estrategia de distribución de la especie está relacionado con los hábitos alimenticios de algunas aves y mamíferos, aunque el comportamiento territorial y migratorio de estos puede introducir complicados elementos en el patrón de distribución espacial.

Se obtuvo mediante la simulación que la población de palmas crecen durante el tiempo establecido en la modelación, por ende, el número de individuos en los diferentes estadíos aumenta según su respectiva tasa de supervivencia y de reproducción, lo que quiere decir que al aumentar el tamaño poblacional de adultos, va aumentar la cantidad de semillas producidas por año, lo cual incrementa la probabilidad de germinación de plántulas, y asimismo, la probabilidad de que estas pasen a los estadíos siguientes (jóvenes y adultos).

La mayor parte de la población de C. quindiuense en la hacienda La Palmera, está conformada por plántulas 639 (individuos), seguido por jóvenes (140 individuos) y adultos (11 individuos). A pesar de que los jóvenes presentaron un valor mayor que adultos, en la simulación realizada en este estadío fue el que descendió más rápida y drásticamente, diezmando su población en menos de un año.

La simulación se realizó además con una variación del $15 \%$, por encima y por debajo de las proba- bilidades de supervivencia y tasa de reproducción. En el caso de la adición del 15\%, se presenta un crecimiento mayor de la población, porque las probabilidades de que se pase de un estadío a otro y de que las semillas germinen, se acrecentará, permitiendo así que la población incremente su potencial reproductivo. Por el contrario, con la sustracción del $15 \%$ sobre las probabilidades, la población de palmas aunque igualmente presenta un aumento en su número de individuos, este no es tan notorio como en el caso anterior. Esto indica que si por algún motivo las tasas de supervivencia y de reproducción decayeran, en 150 años el incremento de población no va a ser significativo comparado con la actual.

Teniendo en cuenta que las poblaciones son dinámicas y que interactúan con otras poblaciones y con el entorno, creando un impacto tanto positivo como negativo sobre estas (Svenning 2001), se modificó la probabilidad de permanencia como adulto (de 1 a 0.80 ), para simular un efecto negativo en la población (enfermedad, incendio, deforestación, ganadería extensiva, entre otros). Una vez realizada esta simulación se obtuvo que si la población continúa con las tasas reales, la población del bosque de la hacienda la palmera se extinguirá en 21 años, siendo en este año que desaparezca el último individuo adulto en este lugar. Por otra parte, si las tasas bajaran $15 \%$, el último adulto permanecería hasta dentro de 19 años y si las tasas aumentaran el mismo 15\%, el último individuo duraría hasta dentro de 22 años.

Lo anterior demuestra la afectación que tiene el impacto antrópico sobre el medio natural, en este caso poblaciones de palma de cera. Entre algunos factores que afectan la dinámica de las poblaciones están la extinción, algunas veces locales de poblaciones de animales, entre ellas aves y mamíferos, que contribuyen a la dispersión de semillas, afectándose así el mecanismo de dispersión (Galeano et al. 2008). Además, factores como la pérdida de poblaciones microbianas que se asocian con la estructura de los suelos los cuales una vez afectados estos modifican de forma negativa el establecimiento y germinación de especies amenazadas (Granville 2008, Svenning 2001), como pudiera ser el caso de C. quindiuense. La sobre-explotación de los suelos es además uno de los factores que inciden en el dinámica poblacional, debido a que los suelos alterados afectan de forma generalizada a todo el eco- 
sistema como consecuencia de su modificación (estructura, nutrientes, capacidad de retención hídrica, etc.) impidiendo que las semillas puedan llevar a cabo su proceso de germinación por falta de luz, nutrientes y espacio, imposibilitando así que los adultos produzcan más semillas y que la viabilidad de estas sea la adecuada (Bernal 2001, Svenning 2001).

La deforestación y fragmentación son además factores característicos de nuestros bosques porque sus zonas de distribución natural se utilizan para el uso agrícola y pecuario. El uso de las tierras para la incursión ganadera es quizás una de las mayores amenazas para las poblaciones de palma porque este tipo de perturbación sumado a que las tasas de sobrevivencia que manejan actualmente las poblaciones en el cambio de un estadío a otro, especialmente de plántulas a juveniles, limitan de una manera considerable el crecimiento y/o mantenimiento de estas poblaciones a través del tiempo (Pintaud et al. 2008).

El mayor índice de mortalidad se observa en el cambio de estadío plántulas-jóvenes, debido a la gran competencia por factores medio ambientales como espacio, nutrientes, luz, etc. En otros estudios demográficos realizados en palmas se ha encontrado que la alta mortalidad está concentrada en plántulas y jóvenes (Olmsted y Álvarez-Buylla 1995, Pintaud et al. 2010). Los riesgos de mortalidad pueden ser por caída de las hojas y ramas, competencia por espacio, luz y nutrientes, efectos de depredación y ataque de plagas (Galindo et al. 2003, Balslev y Baford 1987, Oyama 1990, Salazar y Agudelo 1998).

Si se tienen en cuenta los resultados obtenidos, se encuentra que la palma de cera es una especie que está en peligro de extinción, por lo que es importante implementar programas de conservación para esta especie y su hábitat ya que cumple un papel especialmente importante en los ecosistemas de esta región. Según Pintaud et al. (2008) la protección del hábitat y la cuidadosa gestión de los recursos pueden salvar de la extinción no sólo a esta especie sino a una gran proporción de las diversas especies asociadas con la palma de cera. Con la utilización de las matrices de Leslie podemos decir que $C$. quindiuense es altamente vulnerable por su largo ciclo de vida, lenta germinación y lento crecimiento; necesita de un espacio apropiado para crecer debido a su gran porte y al número y tamaño de las hojas.

A partir de las modelaciones realizadas con las matrices y lo analizado en campo proponemos las principales estrategias de conservación que pudieran reducir su alta vulnerabilidad y mantener su continuidad en el tiempo.

Preservación de los bosques. La naturaleza de la especies de Ceroxylon requiere de la conservación de los bosques montano y pre-montano como un todo (Balick y Beck 1990). Es necesario tomar medidas inmediatas de conservación en aquellas zonas en donde prevalecen relictos de bosque de palma.

El mecanismo más eficaz y eficiente para conservar estos bosques de palma de cera y su biodiversidad, es la concertación participativa con todos los agentes sociales que giran su sustento de la actividad tanto turística como ganadera donde la conservación no sea impuesta como un mecanismo del gobierno local o regional sino un acto de diálogos de saberes donde las diferentes cosmovisiones se acerquen a la no destrucción o degradación del hábitat. Para esto se deben crear incentivos desde el gobierno local y departamental, sobre todo aquellos sitios donde se encuentra relicto de bosque de palma que actualmente está en zona de pastoreo o por medio de la reconversión ganadera. Investigaciones como las realizadas por Girón y Rodríguez (2001) muestran que la implementación de sistemas silvopastoriles permite procesos de regeneración natural y futura consolidación de bosques secundarios, además, se deben entablar acciones e investigaciones enfocadas a entender otros aspectos biológicos y ecológicos de la palma de cera como:

1. La biología reproductiva.

2. Ecología de la dispersión de semillas.

3. Ecología del suelo, para comprender la importancia que tiene el aporte y descomposición de hojarasca en el enriquecimiento de los suelos y la importancia de los organismos asociados (micro y mesofauna, microorganismos) que influyen en los procesos edáficos.

4. Ecología de la fauna asociada y establecer el estatus de «especies claves» para las palmas de cera.

Como conclusión, este estudio comprueba el alto grado de vulnerabilidad que presenta esta especie producto del cómo habitamos el territorio y los usos que le damos al suelo y con las modelaciones estas poblaciones que están empezando entrar en un pro- 
ceso de extinción local debido a que el número de individuos viables está cada vez menor.

\section{Literatura citada}

Álvarez-Buylla ER.1994. Density dependence and pach dynamics in tropical rain forest: matrix models and application to tree species. Am Naturalist. 143: 155-91.

Balslev H, Barfod A. 1987. Ecuadorean palms -an overview. Opera Bot. 92: 17-35.

Balick MJ, Beck H. 1990. Useful palms of the world: a synoptic bibliography. New York: Columbia University Press; 794 p.

Barot S, Gignoux J, Menaut JC. 1999. Demography of a Savanna palm tree: predictions from comprehensive spatial pattern analysis. Ecology. 80: 1987-2005.

Bernal R. 2001. Una nueva especie de Aiphanes (Palmae) de los Andes de Colombia. Caldasia. 23: 163-7.

Borchsenius F, Moraes M. 2006. Diversidad y usos de palmeras Andinas (Arecaceae). Rev Bot Ec Andes Centrales. 2: 41233.

Byt K, Ripley BB. 1980. On sampling paterns by distance methods. Biometrics. 36: 279-89.

Camarero JJ, Rozas V. 2006. Técnicas de análisis espacial de patrones de superficies y detección de fronteras aplicadas en ecología forestal. Invest Agrar: Sist Recur For. 15 (1): 66-87.

Galeano G. 1995. Novedades en el género Ceroxylon (Palmae). Caldasia. 17: 395-408.

Galeano G, Bernal R. 2002. New species and new records of Colombian palms. Caldasia. 24: 277-92.

Galeano G, Sanín MJ, Mejía K, Pintaud JC, Millán B. 2008. Novelties in the genus Ceroxylon (Arecaceae) from Peru, with description of a new species. Rev Per Biol. 15 (1): 65-72.

Galindo R, Betancur J, Cadena J. 2003. Estructura y composición florística de cuatro bosques andinos del Santuario de Flora y Fauna Guanentá-alto río fonce, cordillera Oriental colombiana. Caldasia. 25(2): 313-35.

Gilbert GS, Hubbell SP, Foster RB. 1994. Density and distanceto-adult effects of a canker disease of trees in a moist tropical forest. Oecologia .98: 100-18.

Girón M. 2001. Bosques de Palma de Cera. Armenia: Universidad del Quindío-Pronatta; $252 \mathrm{pp}$.

Girón M, Arboleda M. 2001. Estructura demográfica de la población de Ceroxylon alpinum en el bosque natural de la finca El Cairo, municipio de Salento, Quindío. En: Bosques de Palma de Cera. Armenia: Universidad del Quindío-Pronatta. p. 63-74.

Girón M, Isaza M. Córdoba, MP. 2001. Densidad y patrón espacial de la palma de cera Ceroxylon quindiuense (Karst) H. Wendl. En: Bosques de Palma de Cera. Armenia: Universidad del Quindío-Pronatta. p. 91-103.

Girón M, Rodríguez C. 2001. Plan de acción para la conservación de los bosques de palma de cera Ceroxylon quindiuense y Ceroxylon alpinum, con especial referencia a alto Toche (Tolima) y Cocora (Quindío). En: Bosques de Palma de Cera. Armenia: Universidad del QuindíoPronatta. p. 127-53.

Henderson A, Galeano G. 1996. Euterpe, Prestoea and Neonicholsonia (Palmae). F Neotro Mono. 72: 1-90.
Harper JL. 1977. Population biology of plants. London: London Academic Press; 892 pp.

Harper JL, White J. 1974. The demography of plants. Am Rev Ecol System. 5: 419-63.

Hopkins B. 1954. A new method of determining the type of distribution of plants individuals. Ann Bot. 18: 213-27.

Horvitz CC, Schemeske DW. 1995. Spatiotemporal variation in demographic transitions of a tropical understory herb: projection matrix analysis. Ecol Monog. 65 (2): 155-92.

Koop AL, Horvitz CC. 2005. Projection matrix analysis of the demography an invasive, nonnative shrub (Ardisia elliptica). Ecology. 86 (10): 2661-72.

Krebs CJ. 1978. Ecology: the experimental analysis of distribution and abundance. 2d. ed. New York: Harper \& Row. 320 p.

Mateucci S, Colma A. 1982. Metodología para el estudio de la vegetación. Washington DC: OEA. 167 pp.

Moraes M. 1999. Ecología de palmeras en valles interandinos de Bolivia. Rev Bol Ecol. 5: 3-12.

Moraes M, Galeano G, Bernal R, Balslev H, Henderson A. 1995. Tropical Andean palms. En: Biodiversity and conservation of neotropical Montane Forests. New York: Botanical Garden New York. p. 473-88.

Nieto V, Rodríguez J. 2002. Quercus humboldtii Bonpl. Bogotá: Corporación Nacional de Investigación Forestal.

Odum EP. 1986. Fundamentos de ecología. México, DF: Interamericana. $422 \mathrm{pp}$.

Oliveira-Filho AT, Curi N, Vilela EA, Carvalho DA. 1998. Effects of canopy gaps, topography and soils on the distribution of woody species in a central Brazilian deciduous dry forest. Biotropica. 30: 362-75.

Olmsted G, Álvarez-Buylla ER.1995.Sustainable harvesting of tropical tress: demography and matrix models of two palm species in Mexico. Ecol Applic. 5 (2): 484-500.

Oyama K. 1990. Variation in growth and reproduction in the neotropical dioecious palm Chamedorea tepejilote. $J$ Ecol. 78: 648-63.

Pacala SW, Canham CD, Saponara J, Silander JA, Kobe RK, Ribbens E. 1996. Forest models defined by field measurements: estimation, error analyses, and dynamics. Ecol Monogr. 66: 1-43.

Pintaud JC, Galeano G, Balslev H, et al. 2008. The palms of South America: diversity, distribution and evolutionary history. Rev Per Biol. 15 (1): 7-29.

Pintaud JC, Millan B, Kahn F. 2010. Neotipificación de Ceroxylon weberbaueri Burret. Rev Per Biol. 17 (2): 1636.

Rodríguez-Buriticá S, Orjuela MA, Galeano G. 2005. Demography and life history of Geonoma orbignyana: An understory palm used as foliage in Colombia. Forest Ecol Manag. 211: 329-40.

Salazar GH, Agudelo FD. 1997. Estructura poblacional de palma de cera Ceroxylon quindiuense y Ceroxylon alpinum en Toche (Tolima) y Cocora (Quindío). Trabajo de grado. Programa de Biología y Educación Ambiental. Universidad del Quindío. Armenia, Quindío; $100 \mathrm{p}$.

Spurr SH, Barnes BV. 1980. Ecología forestal. México, DF: AGT editor, SA; 690 pp.

Svenning JC. 2001. On the role of microenvironmental heterogeneity in the ecology and diversification of Neotropical rain-forest palms (Arecaceae). Botanic Rev. 67 (1): 1-53. 